\title{
Development and validation of a multivariable model and online decision-support calculator to aid in preoperative discrimination of benign from malignant splenic masses in dogs
}

\author{
Kristine E. Burgess DVM \\ Lori Lyn Price MAS, MLA \\ Ryan King DVM \\ Manlik Kwong BSEE, BSCS \\ Eliza Grant DVM \\ Katherine A. Olson
}

Jeremiah A. Lyons MVB, PhD

Nicholas A. Robinson BVSc, $\mathrm{PhD}$

Kristin M. Wendelburg DVM

John Berg DVM, MS

From the Department of Clinical Sciences Cummings School of Veterinary Medicine, Tufts University, North Grafton, MA 01536 (Burgess, King, Grant, Olson, Lyons, Robinson, Wendelburg, Berg); Tufts Clinical and Translational Science Institute, Tufts University, Boston, MA 02III (Price); and Institute for Clinical Research and Health Policy Studies, Tufts Medical Center, Boston, MA 02III (Price, Kwong). Dr. Grant's present address is North Country Animal Hospital, North Conway, NH 03860. Dr. Lyons' present address is Regeneron Pharmaceuticals Inc, Tarrytown, NY I059I. Dr. Robinson's present address is bluebird bio Inc, Cambridge, MA 02142. Dr. Wendelburg's present address is Animal Specialty Hospital of Florida, Naples, FL 34II2.

Address correspondence to Dr. Berg (john.berg@ tufts.edu).

\begin{abstract}
OBJECTIVE
To develop a multivariable model and online decision-support calculator to aid in preoperative discrimination of benign from malignant splenic masses in dogs.
\end{abstract}

\section{ANIMALS}

522 dogs that underwent splenectomy because of splenic masses.

\section{PROCEDURES}

A multivariable model was developed with preoperative clinical data obtained retrospectively from the records of 422 dogs that underwent splenectomy. Inclusion criteria were the availability of complete abdominal ultrasonographic examination images and splenic histologic slides or histology reports for review. Variables considered potentially predictive of splenic malignancy were analyzed. A receiver operating characteristic curve was created for the final multivariable model, and area under the curve was calculated. The model was externally validated with data from 100 dogs that underwent splenectomy subsequent to model development and was used to create an online calculator to estimate probability of splenic malignancy in individual dogs.

\section{RESULTS}

The final multivariable model contained 8 clinical variables used to estimate splenic malignancy probability: serum total protein concentration, presence (vs absence) of $\geq 2 \mathrm{nRBCs} / 100 \mathrm{WBC}$, ultrasonographically assessed splenic mass diameter, number of liver nodules $(0, I$, or $\geq 2)$, presence (vs absence) of multiple splenic masses or nodules, moderate to marked splenic mass inhomogeneity, moderate to marked abdominal effusion, and mesenteric, omental, or peritoneal nodules. Areas under the receiver operating characteristic curves for the development and validation populations were 0.80 and 0.78 , respectively.

\section{CONCLUSIONS AND CLINICAL RELEVANCE}

The online calculator (T-STAT.net or T-STAT.org) developed in this study can be used as an aid to estimate the probability of malignancy in dogs with splenic masses and has potential to facilitate owners' decisions regarding splenectomy. ( / Am Vet Med Assoc 2021;258:1362-137I)

\begin{abstract}
S plenic masses in dogs include malignant neoplasms such as hemangiosarcoma, nonendothelial sarcomas, and lymphoma and benign lesions such as hematoma and nodular hyperplasia. ${ }^{1-3}$ Hemangiosarcoma, an aggressive cancer with a high rate of metastasis, is the most common malignant splenic mass. ${ }^{2-5}$ Median survival times reported for dogs with hemangiosarcoma are 19 to 86 days after splenectomy alone and 117 to 277 days after splenectomy with adjuvant treatment. ${ }^{6-14}$ Other malignant splenic masses are less common but are frequently disseminated and ultimately fatal. ${ }^{15,16}$ In contrast, dogs with benign splen-
\end{abstract}

$\begin{array}{ll}\text { ABBREVIATIONS } \\ \text { aPTT } & \text { Activated partial thromboplastin time } \\ \text { FNA } & \text { Fine-needle aspiration } \\ \text { nRBCs } & \text { Nucleated RBCs } \\ \text { PT } & \text { Prothrombin time } \\ \text { ROC } & \text { Receiver operating characteristic } \\ \text { STP } & \text { Serum total protein }\end{array}$

ic masses such as hematoma and nodular hyperplasia have an excellent prognosis after splenectomy.13,15

The decision of whether to elect surgery for a dog with a splenic mass can be difficult for owners because of the disparity in prognosis between dogs with benign and malignant masses and because many dogs are presented with acute signs related to sudden intra-abdominal hemorrhage requiring urgent surgery. Currently, there is no widely available test that allows veterinarians to quickly and reliably distinguish benign from malignant splenic masses soon after a dog is admitted to the hospital. In a study ${ }^{17}$ of 40 dogs with nontraumatic hemoabdomen, hemangiosarcoma-related hemoabdomen could not be reliably differentiated from hemoabdomen due to other causes on the basis of cytologic evaluation of abdominal and peripheral blood smears or assessment of a wide range of routinely measured abdominal fluid and blood analytes. Ultrasound-guided FNA results were shown to be well correlated with core 
needle biopsy results in a study ${ }^{18}$ of 41 dogs with various splenic diseases; however, many of the dogs had diffuse splenomegaly rather than splenic masses, and dogs with hemangiosarcoma, acute hemoabdomen, or both were underrepresented. In dogs with splenic hemangiosarcoma undergoing FNA, hemodilution often complicates cytologic interpretation, ${ }^{19}$ and both FNA and core needle biopsy results may reveal only blood clots and other nonspecific findings. ${ }^{19,20}$ Studies have indicated that increased serum concentrations of the biomarkers thymidine kinase $1^{21}$ and collagen XXVII peptide ${ }^{22}$ have potential to aid in the differentiation of hemangiosarcoma from other splenic masses.

Ultrasonography has appeal as an imaging test with potential to allow discrimination of benign from malignant splenic masses because it is widely available and is routinely used for initial identification of the mass. Splenic hemangiosarcomas typically are inhomogeneous, containing interspersed areas that are anechoic, hypoechoic, and hyperechoic. ${ }^{23}$ Splenic target lesions (masses or nodules with a hypoechoic rim and a hyper- or isoechoic center) are occasionally seen and are frequently, but not consistently, malignant. ${ }^{24}$ Contrast harmonic ultrasonography was not found to be useful for differentiating splenic hemangiosarcoma from hematoma in one study ${ }^{25}$ and was found to have some discriminatory value in another. ${ }^{26}$ Although benign and malignant splenic masses are generally accepted to be indistinguishable with non-contrast-enhanced ultrasonography, ${ }^{25}$ to the best of the authors' knowledge, the use of a combination of ultrasonographic features, including the size and number of splenic masses and their degree of inhomogeneity, has not been investigated for this purpose in a large number of dogs.

The lack of laboratory and imaging tests that can reliably distinguish benign from malignant masses has led clinicians to rely on broad guidelines that pertain to the general population of dogs with splenic masses. One of these is described as the law of two-thirds: approximately two-thirds of dogs with a splenic mass have a malignant tumor, and approximately two-thirds of malignant splenic tumors are hemangiosarcomas. ${ }^{20}$ Similarly, retrospective investigations have indicated that $63 \%^{27}$ and $70 \%{ }^{28}$ of dogs with nontraumatic hemoabdomen have splenic hemangiosarcoma, and $76 \%$ of dogs with splenic masses that cause hemoabdomen requiring transfusion have hemangiosarcoma. ${ }^{29}$ It is also known that benign splenic masses are typically larger than malignant masses at the time of diagnosis. ${ }^{30}$ Although these observations are important, they have limited value in predicting the type of splenic mass for individual dogs with various signalments, clinical signs, and laboratory and diagnostic imaging findings. The limitation is particularly concerning because owners of dogs with splenic masses are often faced with a choice between surgery and euthanasia, and reliance on broad probabilities may result in decisions to elect euthanasia for dogs with a curable disease.

In human medicine, a common solution to the problem of risk assessment and prognostication for individual patients is the development of online clinical decision-support calculators that can estimate the probabilities of various clinical outcomes such as having a given disease or experiencing a certain response to treatment. ${ }^{31,32}$ These calculators are developed through multivariable analyses of potential predictive variables selected by clinicians using data from a large series of patients with known outcomes and can facilitate decision-making for both clinicians and patients. Recently, a decision-support model was developed for estimating a risk score for hemangiosarcoma in dogs with nontraumatic hemoabdomen. ${ }^{33}$ Variables included in the model were body weight, plasma total protein concentration, platelet count, and thoracic radiographic findings. Areas under the ROC curve (a measure of the discriminatory accuracy of predictive models) for the model development and external validation populations in that study ${ }^{33}$ were 0.85 and 0.77 , respectively.

The purpose of the study reported here was to develop and validate a multivariable model and online decision-support calculator that could be used to estimate the probability of malignancy in individual dogs with splenic masses on the basis of preoperative variables obtained for a large number of dogs with known diagnoses. Our hypothesis was that a model with a degree of accuracy adequate to aid in clinical decisionmaking could be developed.

\section{Materials and Methods}

\section{Case selection}

Electronic medical records searches were conducted to identify dogs that underwent splenectomy for a splenic mass at the Foster Hospital for Small Animals, Cummings School of Veterinary Medicine, Tufts University, or at TuftsVETS, a satellite referral hospital. A non-commercially available electronic medical records system was used before June 1, 2014, and a commercially available system ${ }^{\mathrm{a}}$ was used after that time.

For purposes of model development, the medical records from August 1, 2001, to July 31, 2012, were searched. The search terms included splenectomy and hemangiosarcoma. Dogs were included in this part of the study if images obtained during preoperative abdominal ultrasonographic examination and histologic slides or a histology report for the excised splenic mass were available for review.

\section{Medical records review}

Preoperative data collected from the medical records included signalment, body weight, clinical signs, physical examination findings, results of laboratory tests (CBC, serum biochemical analysis, measurements of serum lactate concentration before and after fluid resuscitation, coagulation profiles, and thromboelastography) and results of thoracic radiography and abdominal ultrasonography. Laboratory results were considered only if they were performed at admission or $\leq 30$ days before this date; when sequential results were recorded, the values obtained closest to the time of splenectomy were used. Prothrombin time and aPTT were measured in citrated whole blood with a handheld device ${ }^{\mathrm{b}}$ or in noncitrated samples with a laboratory-based device. ${ }^{c}$ The 
reference intervals for the hand-held device (PT, 11 to 17 seconds; aPTT, 72 to 102 seconds) were considerably wider than those for the laboratory-based device (PT, 6.2 to 9.3 seconds; aPTT, 8.9 to 16.3 seconds). To adjust for this, coagulation times in seconds were converted to percentages of the upper limit of the respective reference intervals.

\section{Diagnostic imaging}

A board-certified veterinary radiologist (RK), who was blinded to the histologic diagnoses of the splenic masses, retrospectively evaluated ultrasonographic variables by use of recorded still images and cineloops. All images had been obtained with dogs gently restrained in a position as close to dorsal recumbency as possible. If the recorded images were not sufficient for assessing an individual variable (eg, when the entire splenic mass was not included in an image), imaging reports were reviewed. If a variable could not be assessed by review of the images or imaging reports, the dog was excluded from the study. Data collected included severity of any abdominal effusion; diameter and number of splenic masses; severity of any splenic mass inhomogeneity; presence of 0,1 , or $\geq 2$ liver nodules; and presence or absence of nodules in the mesentery, omentum, or peritoneum.

Abdominal effusion was subjectively categorized on the basis of volume as none, mild, moderate, or marked (Figure I). Dogs considered to have mild abdominal effusion had small, hypoechoic to anechoic triangular foci extending around visceral organs. Dogs considered to have moderate effusion had larger, yet isolated, approximately triangular foci separating visceral organ structures. In dogs considered to have marked effusion, visceral structures and mesentery floated in and were surrounded by hypoechoic to anechoic regions.

The largest measurable diameter of the splenic mass was determined. When multiple splenic masses were present, diameter and inhomogeneity of the largest mass were assessed. To account for the possible influence of the relationship between patient size and mass size, the ratio of mass diameter (in centimeters) to body weight (in kilograms) was calculated. Splenic

mass inhomogeneity was subjectively categorized as none, mild, moderate, or marked (Figure 2). The most inhomogeneous region of each mass was used for the inhomogeneity assessment. Dogs considered to
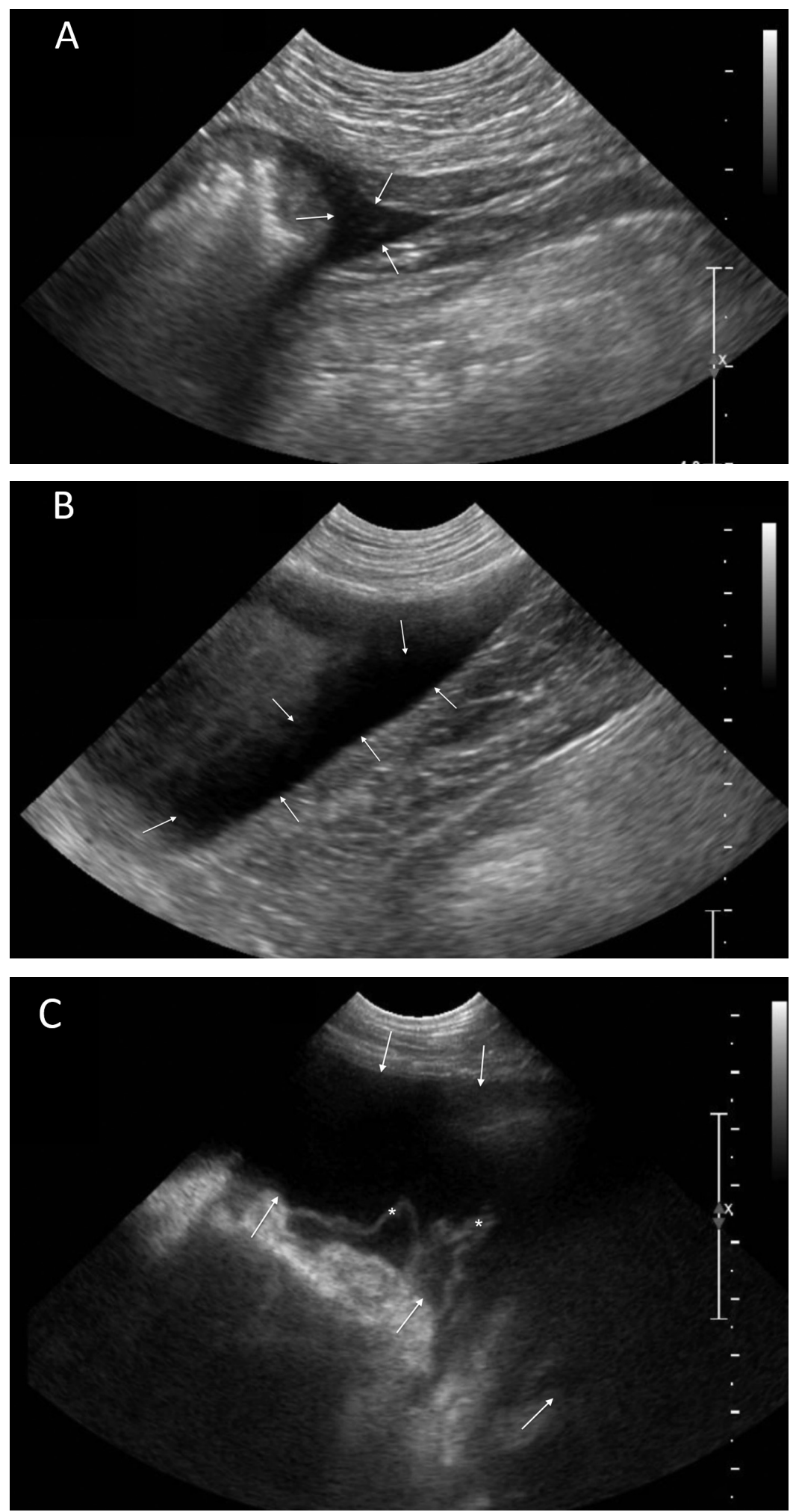

Figure I-Representative preoperative ultrasonographic images showing abdominal effusion (arrows) subjectively categorized as mild (A), moderate (B), and marked (C) in dogs that underwent splenectomy. Notice the mesentery (asterisks) floating within the effusion in panel $\mathrm{C}$. Dashed lines in the scale bars to the right of the images represent distance in $\mathrm{I}-\mathrm{cm}$ increments. have mild splenic mass inhomogeneity had regions of mildly mixed echogenicity, including regional alterations in echotexture. Dogs considered to have moderate splenic mass inhomogeneity had more disparity 

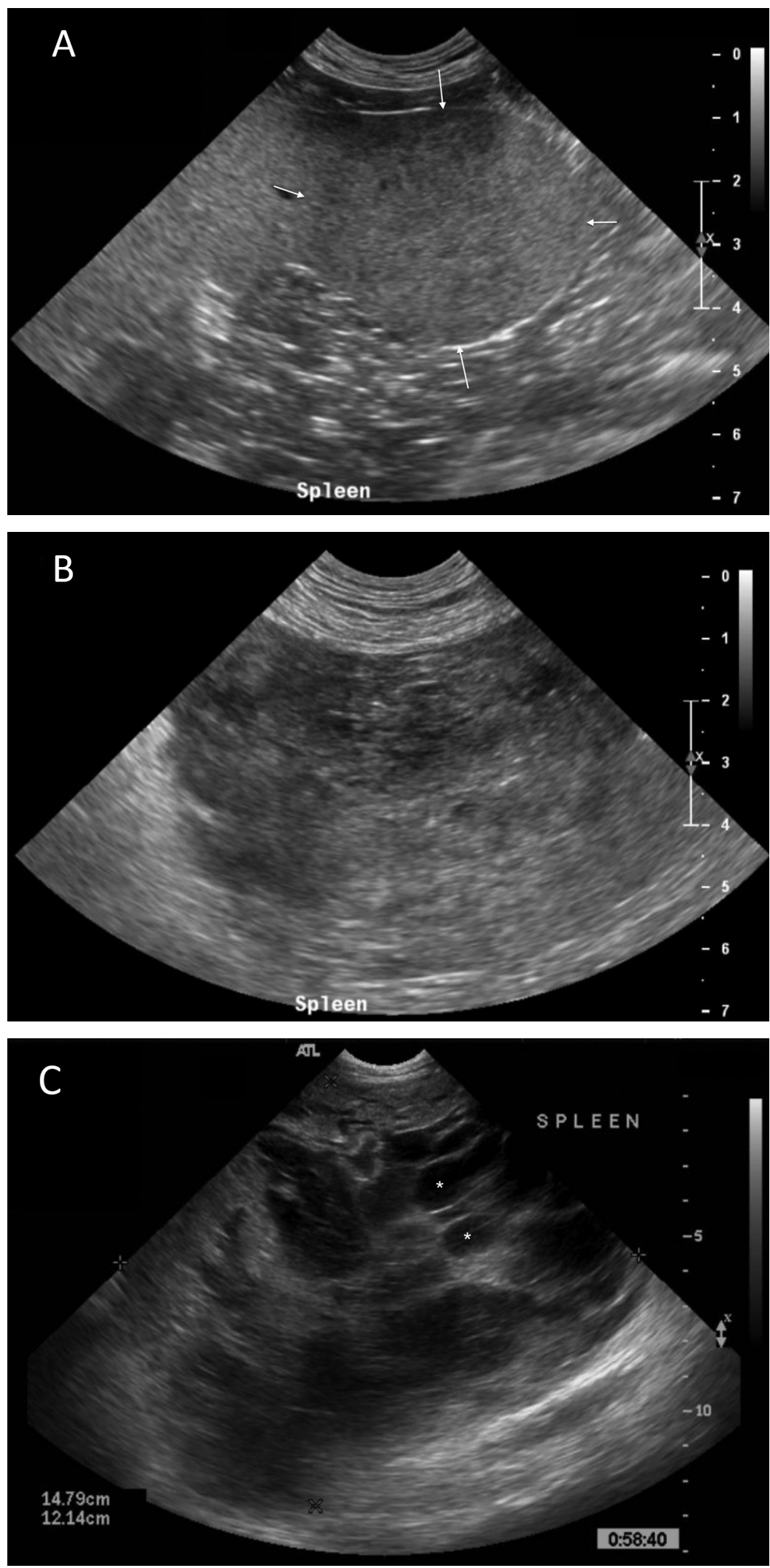

Figure 2-Representative preoperative ultrasonographic images of splenic masses showing inhomogeneity subjectively categorized as mild (A), moderate (B), and marked (C) in dogs that underwent splenectomy. A-Regions of mildly mixed echogenicity are present in the splenic mass (arrows). B-There is greater disparity in echotexture, compared with the mass in panel $\mathrm{A}$; the mass effaces normal splenic architecture. $\mathrm{C}$ The mass effaces normal splenic architecture with marked disparity between hypo- and hyperechoic regions, and cavitations, a feature of marked inhomogeneity, are present (asterisks). See Figure I for remainder of key. between normal and affected regions, often with mixed regions of hypo- and hyperechogenicity. Dogs considered to have marked inhomogeneity had marked disparity between hypoechoic and hyperechoic regions and often had effacement of all normal parenchymal architecture in the affected regions, foci of cavitation, or both.

\section{Histologic evaluation}

One veterinary pathologist (JAL) reviewed all available histologic slides. When slides were unavailable for review, the initial histologic diagnosis was obtained from the medical records. For dogs with an initial diagnosis of undifferentiated sarcoma, immunohistochemical testing for factor VIII-related antigen $^{\mathrm{d}}$ was performed, and factor VIII-positive tumors were recategorized as hemangiosarcoma. ${ }^{34}$ Dogs with grade 1 or 2 fibrohistiocytic nodules were classified as having benign disease, and dogs with grade 3 fibrohistiocytic nodules were classified as having malignant disease. Grading was performed on the basis of the lymphoid-tofibrohistiocytic cell ratio as previously described. ${ }^{35}$

\section{Statistical analysis}

Multivariable model developmentVariables considered to have potential to be predictive of splenic malignancy were selected prior to statistical analysis. Variables for which the preponderance of results were the same (eg, results of thoracic radiography for pulmonary metastases) and variables that could not consistently be accurately assessed through a review of medical records (eg, the nature and duration of clinical signs directly related to the splenic mass) were not examined.

Univariable logistic regression analysis $^{\mathrm{e}}$ was performed to estimate the strength of associations between the selected variables and the presence of a malignant splenic mass. To determine whether relationships between continuous variables and malignancy were linear or nonlinear, smoothed splines depicting the relationship between each variable and likelihood of malignancy were created. The splines were examined for linearity by use of restricted cubic spline regression. ${ }^{f}$ For continuous variables that had nonlinear relationships with malignancy, if the smoothed spline contained a single inflection point, piecewise linear regression was performed with a cutpoint 
corresponding to the inflection point. To simplify multivariable analysis when the smoothed spline contained $>1$ inflection point, the variable was converted to a categorical variable with cutpoints corresponding to the inflection points.

Variables that had values of $P \leq 0.20$ in the univariable analysis for association with malignancy were considered for multivariable regression analysis. Variables for which values were not available for $\geq$ 95\% of the dogs were omitted from this analysis. Backward stepwise regression analysis was used, and all variables with a value of $P<0.05$ were included in the final model. An ROC curve was created for the final model, and the area under the curve was calculated and used to assess the ability of the model to discriminate benign from malignant splenic masses. Mean estimated probabilities of malignancy were calculated for dogs with histologically confirmed benign and malignant tumors. Diagnostics, including examination of deviance residuals and measurements of the effects of deleting each variable on the remaining regression coefficients (ie, DFBETA statistics), were performed to determine whether the final model was unduly influenced by outlying data. Estimated probabilities produced by the decision-support calculator being developed were checked against probabilities produced by the statistical software. ${ }^{\mathrm{e}}$

External model validation-Subsequent to model development, 100 additional dogs that underwent splenectomy for splenic masses between June 1, 2015, and November 30, 2018, were identified through a search of electronic medical records. Search terms included splenectomy and hemangiosarcoma. Dogs were included in the validation population if they had values for all variables included in the final multivariable model and if histologic slides or a histology report for the excised splenic mass were available. One board-certified veterinary radiologist $(\mathrm{RK})$, blinded to the histologic diagnoses of the splenic masses, reviewed all available ultrasonographic images, and a board-certified pathologist (NAR) reviewed all available histologic slides as described for dogs in the model development portion of the study. The $\beta$ coefficients (indicators of the strength and direction of the relationships between the predictor variables and an outcome of malignancy) from the model were applied to the data set for the validation population, an ROC curve was generated, and the area under the ROC curve was determined. ${ }^{\text {e }}$ The final model was used to develop an online decision-support calculator that can be used by veterinarians to quickly estimate the probability of malignancy for individual dogs.

\section{Results}

A total of 422 dogs that met the study inclusion criteria were used for model development. Twenty-three dogs were excluded because abdominal ultrasono-

Table I-Descriptive statistics for variables initially considered potentially predictive of splenic malignancy in a study to develop a multivariable model and online decision-support calculator to aid in preoperative discrimination of benign from malignant splenic masses in dogs.

\begin{tabular}{|c|c|c|c|}
\hline Variable & No. of dogs* & $\begin{array}{l}\text { Dogs with benign } \\
\text { masses }(n=193)\end{array}$ & $\begin{array}{l}\text { Dogs with malignant } \\
\text { masses }(n=229)\end{array}$ \\
\hline \multicolumn{4}{|l|}{ Continuous data } \\
\hline Age $(y)$ & 422 & $10.6 \pm 2.2$ & $10.2 \pm 2.3$ \\
\hline Body weight (kg) & 415 & $29.2 \pm 13.4$ & $30.8 \pm 11.6$ \\
\hline PCV (\%) & 418 & $37.5 \pm 9.7$ & $32.9 \pm 9.0$ \\
\hline PT $(\%) \dagger$ & 167 & $0.9 \pm 0.2$ & $1.0 \pm 0.5$ \\
\hline aPTT $(\%) \dagger$ & 165 & $1.1 \pm 0.9$ & $1.0 \pm 0.4$ \\
\hline Serum lactate $(\mathrm{mmol} / \mathrm{L})$ & 315 & $3.2 \pm 2.4$ & $4.5 \pm 3.3$ \\
\hline Platelet count (X I,000/ $/ \mu \mathrm{L})$ & 366 & $248.5 \pm 160.6$ & $167.3 \pm 138.8$ \\
\hline STP $(g / d L)$ & 417 & $6.7 \pm 1.1$ & $6.1 \pm 1.2$ \\
\hline Splenic mass diameter $(\mathrm{cm})$ & 410 & $8.8 \pm 5.9$ & $7.8 \pm 4.6$ \\
\hline Splenic mass diameter-to-body weight ratio $(\mathrm{cm} / \mathrm{kg})$ & 402 & $0.4 \pm 0.4$ & $0.3 \pm 0.3$ \\
\hline \multicolumn{4}{|l|}{ Categorical data } \\
\hline Acanthocytes $\ddagger$ & $52 / 368$ & $31(17.9)$ & $21(10.8)$ \\
\hline$\geq 2$ nRBCs/I00 WBCs $\ddagger$ & $83 / 416$ & $21(10.9)$ & $62(27.7)$ \\
\hline Schistocytes $\ddagger$ & $37 / 368$ & $24(12.3)$ & $13(7.5)$ \\
\hline Moderate or marked abdominal effusion $\ddagger$ & |44/422 & $34(17.6)$ & $110(48.0)$ \\
\hline Multiple splenic masses or nodules $\ddagger$ & $|65 / 4| 6$ & $50(26.2)$ & $115(5 \mathrm{I} .1)$ \\
\hline Moderate or marked splenic mass inhomogeneity $\ddagger$ & $332 / 4 \mid 3$ & $132(69.8)$ & $200(89.3)$ \\
\hline Splenic cavitations $\ddagger$ & 107/177 & $43(57.3)$ & $64(62.8)$ \\
\hline \multicolumn{4}{|l|}{ No. of liver nodules } \\
\hline 0 & $333 / 422$ & $163(84.4)$ & I $70(74.3)$ \\
\hline I & $30 / 422$ & $10(5.2)$ & $20(8.7)$ \\
\hline$\geq 2$ & $59 / 422$ & $20(10.4)$ & $39(17.0)$ \\
\hline Mesenteric, omental, or peritoneal nodules $\ddagger$ & $37 / 417$ & $8(4.2)$ & $29(12.9)$ \\
\hline
\end{tabular}

Data reflect preoperative findings collected retrospectively from the medical records (including retrospective review of ultrasonographic images and available histologic slides) of 422 dogs that were used for model development. Results are reported as mean \pm SD (continuous variables) or number (\%) of dogs (categorical variables).

*For continuous data, values in this column indicate the number of dogs with data available. For categorical data, the proportion of dogs with data available that had a positive result for the category is shown. †Because different instruments were used to measure the same variable, values are reported as the percentage of the upper limit of the applicable reference interval. $\ddagger$ Recorded as present (vs absent). 
graphic images were not available, and 9 dogs were excluded because histologic slides or reports were not available. The mean \pm SD age and body weight of the 422 dogs were $10.4 \pm 2.26$ years and $30.11 \pm$ $12.47 \mathrm{~kg}(66.24 \pm 27.43 \mathrm{lb})$, respectively. More than 50 breeds were represented, with Labrador Retrievers (n = 45), Golden Retrievers (41), and German Shepherd Dogs (28) most commonly included; there were also 79 mixed-breed dogs. There were 22 sexually intact males, 207 neutered males, 9 sexually intact females and 184 neutered females.

Histologic slides were available for review for 421 of 422 dogs. The dog that did not have slides available had a diagnosis of hemangiosarcoma. Two hundred twentynine of 422 (54.3\%) dogs had malignant splenic masses, and 193 (45.7\%) dogs had benign splenic masses. Of 17 malignant masses initially diagnosed as undifferentiated sarcoma, 2 tested positive for factor VIII-related antigen on immunohistochemical testing and were reclassified as hemangiosarcoma. Hemangiosarcoma was the most common malignant mass, diagnosed in 165 of 422 (39.1\%) dogs overall and in 165 of 229 (72.1\%) dogs with malignant masses. Other malignant masses were lymphoma $(n=20)$, histiocytic sarcoma $(11)$, undifferentiated neoplasms (15), grade 3 fibrohistiocytic nodules (10), soft tissue sarcomas (4), and unspecified round cell tumor, leiomyosarcoma, metastatic adenocarcinoma, and osteosarcoma (1 each). Splenic hematoma was the most common benign mass and was diagnosed in 88 of 422 (20.9\%) dogs overall and in 88 of 193 (45.6\%) dogs with benign masses. Seventy-eight of 193 (40.4\%) dogs with benign masses had a diagnosis of lymphoid hyperplasia. Other benign masses were diagnosed as grade 1 or 2 fibrohistiocytic nodules $(n=6)$, extramedullary hematopoiesis (6), myelolipoma (4), leiomyoma (1), and other benign or nonneoplastic conditions (10).

Ultrasonography variables were determined solely on the basis of a retrospective review of the available images for 402 of 422 (95.3\%) dogs. Written records were used to aid assessment of at least one of these variables for 20 (4.7\%) dogs.

Descriptive statistics and results of univariable analysis for the variables initially considered to be potentially predictive of splenic malignancy were summarized (Tables I and 2). Among the continuous variables, age and splenic mass diameter were found to have nonlinear relationships with malignancy with single inflection points. However, because the nonlinearity of the relationship between age and malignancy was highly influenced by a small number of very young and very old dogs, age was treated as a linear variable. For splenic mass diameter, a cutpoint of $7 \mathrm{~cm}$ was chosen for piecewise linear regression. The splenic mass diameterto-body weight ratio and number of liver nodules had

Table 2-Results of univariable analysis for variables initially considered to be potentially predictive of splenic malignancy for the same dogs as in Table I.

\begin{tabular}{|c|c|c|c|}
\hline Variable & $\begin{array}{c}\text { No. of dogs } \\
\text { with data available }\end{array}$ & OR $(95 \% \mathrm{CI})$ & $P$ value \\
\hline \multicolumn{4}{|l|}{ Continuous data } \\
\hline Age (y) & 422 & $0.93(0.85-1.01)$ & 0.09 \\
\hline Body weight $(\mathrm{kg})$ & 415 & $1.01(0.995-1.03)$ & 0.20 \\
\hline PCV (\%) & 418 & $0.95(0.93-0.97)$ & $<0.001$ \\
\hline PT $(\%) \dagger$ & 167 & $3.41(0.92-12.72)$ & 0.07 \\
\hline aPTT $(\%) \dagger$ & 165 & $0.74 \mid(0.43-I .27)$ & 0.28 \\
\hline Serum lactate $(\mathrm{mmol} / \mathrm{L})$ & 315 & $1.19(1.08-1.30)$ & $<0.001$ \\
\hline Platelet count $(X 1,000 / \mu \mathrm{L})$ & 366 & $0.996(0.995-0.998)$ & $<0.001$ \\
\hline STP $(\mu g / d L)$ & 417 & $0.60(0.50-0.72)$ & $<0.001$ \\
\hline Splenic mass diameter $(\mathrm{cm}) \S$ & 410 & & \\
\hline$\leq 7$ & & $1.27(1.12-1.44)$ & $<0.001$ \\
\hline$>7$ & & $0.87(0.82-0.93)$ & $<0.001$ \\
\hline \multicolumn{4}{|l|}{ Categorical data } \\
\hline Acanthocytes $\ddagger$ & 368 & $0.55(0.30-1.00)$ & 0.052 \\
\hline$\geq 2 \mathrm{nRBCs} / 100 \mathrm{WBCs} \ddagger$ & 416 & $3.12(1.82-5.35)$ & $<0.001$ \\
\hline Schistocytes $\ddagger$ & 368 & $1.73(0.85-3.15)$ & 0.13 \\
\hline Moderate or marked abdominal effusion $\ddagger$ & 422 & $4.32(2.75-6.79)$ & $<0.001$ \\
\hline Splenic mass diameter-to-body weight ratio $(\mathrm{cm} / \mathrm{kg})$ & 402 & & \\
\hline$\geq 0.4$ & & - & - \\
\hline 0.2 to $<0.4$ & & $1.97(1.91-3.26)$ & 0.008 \\
\hline$<0.2$ & & $2.04(1.25-3.33)$ & 0.004 \\
\hline Multiple splenic masses or nodules $\ddagger$ & 416 & $2.95(1.95-4.47)$ & $<0.001$ \\
\hline Moderate or marked splenic mass inhomogeneity $\ddagger$ & 413 & $3.60(2.13-6.08)$ & $<0.001$ \\
\hline Splenic cavitations $\ddagger$ & 177 & $1.25(0.68-2.30)$ & 0.47 \\
\hline No. of liver nodules & 422 & & \\
\hline 0 & & - & - \\
\hline I & & $1.92(0.87-4.22)$ & 0.106 \\
\hline$\geq 2$ & & $1.87(1.05-3.34)$ & 0.035 \\
\hline Mesenteric, omental, or peritoneal nodulesł & 417 & $3.40(1.52-7.64)$ & 0.003 \\
\hline
\end{tabular}

Odds ratios $>$ I or $<1$ indicate an increase or decrease in odds of malignancy, respectively. For continuous variables, ORs indicate the change in odds with each incremental (I-unit) increase in the value of the variable. For categorical variables, ORs indicate the change in odds when the variable is present (vs absent).

$\S$ Analyzed with piecewise linear regression owing to a nonlinear relationship with malignancy with a single inflection point. $-=$ Not applicable (referent category).

See Table I for remainder of key. 
nonlinear relationships with splenic malignancy, with 2 inflection points each, and were converted to categorical variables. The remaining continuous variables had linear relationships with malignancy.

Among the categorical variables, abdominal effusion and splenic mass inhomogeneity (initially classified as none, mild, moderate, or marked) were each collapsed into 2 categories. For abdominal effusion, although the OR for patients in the none versus mild categories was significant $(1.67 ; 95 \% \mathrm{CI}, 1.03$ to $2.72 ; P=$ $0.04)$, the OR for the moderate versus marked categories was not $(0.50 ; 95 \% \mathrm{CI}, 0.22$ to $1.12, P=0.09)$. Only 6 dogs had no splenic mass inhomogeneity, and the OR for patients in the moderate versus marked categories for this variable was nonsignificant $(0.7 ; 95 \% \mathrm{CI}, 0.46$ to $1.12 ; P=0.14)$. For these reasons, and because it would simplify selection of categories by users of the decision-support calculator being developed, the categories of none and mild and the categories of moderate and marked were combined for the abdominal effusion and splenic mass inhomogeneity variables, and these data were reanalyzed (Table 2).

The following variables that had values of $P \leq 0.20$ in the univariable analysis were omitted from multivariable analysis because values were not available for $\geq 400$ patients: PT, serum lactate concentration, platelet count, presence of acanthocytes, and presence of schistocytes. The final candidate variables for multivariable analysis were age, body weight, PCV, STP concentration, splenic mass diameter, splenic mass diameter-to-body weight ratio, number of liver nodules, and presence (vs absence) of $\geq 2 \mathrm{nRBCs} / 100 \mathrm{WBCs}$, moderate or marked abdominal

Table 3-Results of the final multivariable model used as the basis for an online decision-support calculator that was developed to aid in preoperative discrimination of benign from malignant splenic masses in dogs.

\begin{tabular}{|c|c|c|c|}
\hline Variable & $\beta$ Coefficient & OR $(95 \% \mathrm{Cl})$ & $P$ value \\
\hline Intercept & -1.45 & & \\
\hline STP $(\mathrm{mg} / \mathrm{dL})$ & -0.22 & $0.80(0.65-1.00)$ & 0.045 \\
\hline$\geq 2$ nRBCs//100 WBCs $\ddagger$ & 0.85 & $2.33(1.23-4.4 I)$ & 0.009 \\
\hline Moderate or marked abdominal effusion $\ddagger$ & I.II & $3.02(\mid .72-5.31)$ & $<0.001$ \\
\hline \multicolumn{4}{|l|}{ Splenic mass diameter $(\mathrm{cm}) \S$} \\
\hline$\leq 7$ & 0.22 & $1.24(1.07-1.45)$ & 0.006 \\
\hline$>7$ & -0.09 & $0.91(0.86-0.98)$ & 0.008 \\
\hline Multiple splenic masses or nodules $\ddagger$ & 1.17 & $3.21(1.89-5.44)$ & $<0.001$ \\
\hline Moderate or marked splenic mass inhomogeneitył & 1.06 & $2.89(1.53-5.48)$ & 0.001 \\
\hline No. of liver nodules & & & 0.029 \\
\hline I & 0.78 & $2.19(0.86-5.56)$ & 0.10 \\
\hline$\geq 2$ & 0.82 & $2.28(|| 2-4.6 \mid)$. & 0.02 \\
\hline Mesenteric, omental, or peritoneal nodulesł & 0.94 & $2.55(1.04-6.27)$ & 0.04 \\
\hline
\end{tabular}

The final model included data from 390 dogs that had results for all variables shown. The $\beta$ coefficient indicates the strength and direction of the relationship between the variable shown and an outcome of malignancy.

See Tables I and 2 for remainder of key.
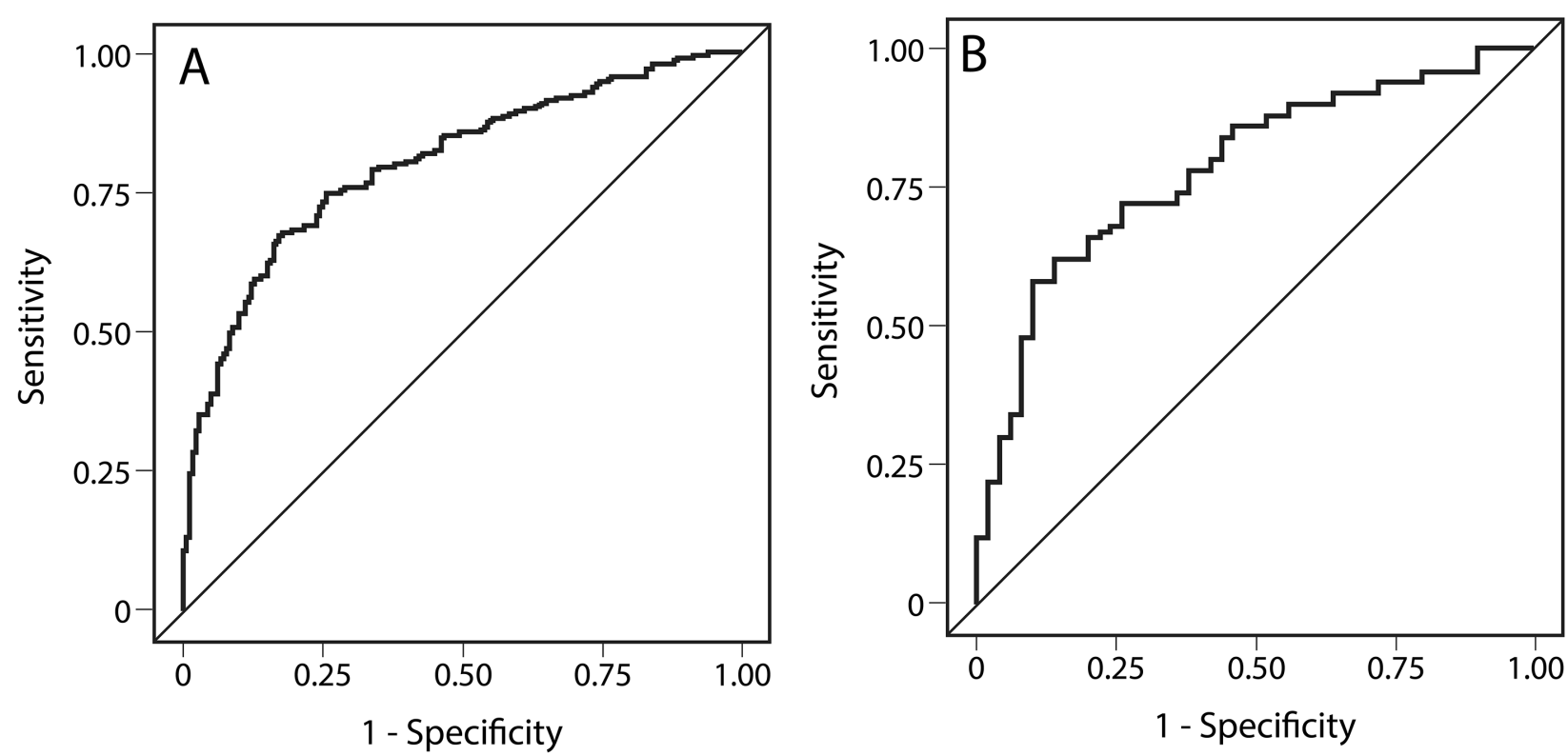

Figure 3-Receiver operating characteristic curves of the final multivariable model for the development $(A$; area under the ROC curve, 0.80 ) and validation $(B ; 0.78)$ data sets. The development and validation data sets contained 422 and 100 dogs that underwent splenectomy, respectively. The $45^{\circ}$ diagonal line represents the line of no discrimination. See Table 3 for variables included in the final model. 
effusion, multiple splenic masses or nodules, moderate or marked splenic mass inhomogeneity, and mesenteric, omental, or peritoneal nodules. The multivariable analysis included data on 390 dogs for which results were available for all of these variables and produced the final model shown (Table 3). The area under the ROC curve for the final model was 0.80 (95\% CI, 0.76 to 0.85 ; Figure 3). The mean \pm SD estimated probability of malignancy yielded by the decision-support calculator was $39 \pm 21 \%$ (95\% CI, 36\% to $42 \%$ ) and $66 \pm 23 \%$ (95\% CI, 63\% to $70 \%$ ) for dogs that had histologically confirmed benign tumors and histologically confirmed malignant tumors, respectively. Results of model diagnostic testing indicated that the model was not unduly influenced by outlying data. When results produced by the calculator were checked against results produced by the statistics software program, the difference in predicted probabilities ranged from $0.0 \%$ to $0.003 \%$.

The population used in the external model validation comprised 50 dogs with malignant tumors and 50 dogs with benign tumors. Among the malignant tumors, 35 (70\%) were hemangiosarcomas. The area under the ROC curve for the final model developed with data from the validation population was 0.78 (95\% CI, 0.69 to 0.87 ; Figure 3).

The final model was used to develop an online decision-support calculator (available at T-STAT.net or T-STAT. org). ${ }^{\mathrm{g}}$ Details regarding the calculation procedure and an example calculation of the probability of malignancy based on data from a hypothetical dog are available (Supplementary Appendix SI, available at: avmajournals. avma.org/doi/suppl/10.2460/javma.258.12.1362).

\section{Discussion}

The online decision-support calculator described in this report was developed to assist veterinarians in advising owners regarding the probability of malignancy in individual dogs with splenic masses and is based on data that are commonly collected soon after admission. The calculator may facilitate owner decisions to elect splenectomy or not and has potential to reduce the risk that an owner will erroneously elect euthanasia for a dog with a benign mass that could have been cured by surgical removal. The area under the ROC curve for the final statistical model was 0.80, indicating that for any randomly chosen pair of dogs, one with a malignant splenic mass and the other with a benign splenic mass, the probability that the model will assign a higher probability of malignancy to the dog with the malignant mass is $80 \% .{ }^{36}$ For medical tests, the area under the ROC curve in the ranges of 0.7 to $<0.8,0.8$ to $<0.9$, and $\geq 0.9$ are considered to indicate acceptable, excellent, and outstanding discriminatory ability, respectively. ${ }^{36}$ Although the area under the ROC curve for the model confirmed our hypothesis that a model with an accuracy level adequate to assist in clinical decision-making could be developed, the probabilities of malignancy estimated by the calculator should always be considered as supplementary to the full clinical picture. For example, calculator results that do not indicate a high probability of malignancy should not supersede clear clinical evidence that a splenic mass is malignant, such as radio- graphic evidence of pulmonary metastases or echocardiographic evidence of a concurrent right atrial mass.

The finding that STP concentration and the presence of moderate or marked abdominal effusion were independently associated with malignancy suggested that factors other than the degree of blood loss may have influenced STP results. For example, it is known that albumin, which accounts for $35 \%$ to $50 \%$ of STP, is a negative acute-phase protein that can decrease in response to inflammation of any cause, including cancer. ${ }^{37}$ It has been shown that dogs with mammary tumors and clinically detectable metastases have significantly decreased serum albumin concentrations, compared with dogs that have mammary tumors without metastases, ${ }^{38}$ and that several positive acute-phase proteins (produced in response to inflammation) are known to be variably increased in the serum of dogs with mammary carcinoma and other cancers. ${ }^{39}$

A possible relationship between splenic hemangiosarcoma and the presence of abnormal circulating RBCs, including acanthocytes, schistocytes, and nRBCs, has long been recognized. ${ }^{20}$ Acanthocytes and schistocytes likely result from mechanical fragmentation within the abnormal vasculature of the splenic mass, ${ }^{17,20,40}$ and because these cells are normally removed from the circulation by the spleen, they may tend to accumulate in the blood of patients with destruction of substantial amounts of normal splenic tissue. ${ }^{17,40}$ Although acanthocytes ${ }^{17,41,42}$ and schistocytes are occasionally observed in dogs with hemangiosarcoma, ${ }^{1,43}$ they are also frequently present in dogs with nonneoplastic diseases that can cause RBC fragmentation ${ }^{17,41-43}$ and therefore have limited value as an aid in determining the presence of hemangiosarcoma. Nucleated RBCs are circulating immature erythrocytes that can be associated with anemia of any cause. Consistent with the tendency of splenic hemangiosarcomas to rupture and bleed, we found that increased circulating concentrations of $\mathrm{nRBCs}(\geq 2 / 100 \mathrm{WBCs})$ were an independent predictor of malignancy. This proportion was considered abnormal in our study because isolated nRBCs can be occasionally observed in samples from healthy dogs and were considered an incidental finding by our laboratory.

Six of the variables included in our model must be assessed through a complete ultrasonographic examination of the abdomen. Our finding of an association between moderate or marked abdominal effusion and malignancy suggested that although benign masses are occasionally associated with hemoabdomen, ${ }^{30}$ they may produce less severe blood loss than hemangiosarcoma. The observation that benign splenic masses may become significantly larger than hemangiosarcomas ${ }^{30}$ suggests that benign masses grow relatively slowly, allowing the patient to compensate, whereas hemangiosarcomas tend to rupture early in their growth, producing clinical signs. Our results indicated that very small splenic nodules were commonly benign and the probability of malignancy increased as mass diameter approached about $7 \mathrm{~cm}$; when the largest diameter of the splenic mass (or largest splenic mass in patients with $>1$ observed) exceeded $7 \mathrm{~cm}$, masses of increasing size were progressively less likely to be malignant. We considered it possible that the $7-\mathrm{cm}$ cutpoint may have represented an approximate size by which most hemangiosarcomas have ruptured or are at imminent risk of rupturing. 
To our knowledge, associations between malignancy and the presence of multiple splenic masses or moderate or marked splenic mass inhomogeneity have not been previously reported. In a previous study ${ }^{44}$ of 31 dogs in which splenic mass lesions were categorized as solitary or multiple on the basis of ultrasonographic findings, no association between multiple lesions and malignancy was identified. We considered it likely that malignant masses would have greater inhomogeneity than benign masses because of the variable echogenicity of cancerous tissue and interspersed regions of clotted blood and serosanguinous fluid. A limitation of the model developed in this study is that ultrasonographic characterization of the severity of abdominal effusion and splenic mass inhomogeneity is inherently subjective and operator dependent. However, we found that both variables could be stratified into 2 easily distinguished categories (none or mild and moderate or marked) without compromising the variables' value when discriminating between benign and malignant masses. The website for the online calculator provides example images for each category. If an owner's first priority is to avoid the mistake of electing euthanasia for a dog with benign disease and if mild to moderate mass inhomogeneity or abdominal effusion is present but the appropriate category to select is unclear, selection of the none or mild category by the user will produce a more conservative estimate of the probability of malignancy. The findings that the number of liver nodules and presence of mesenteric, omental, or peritoneal nodules were associated with splenic malignancy were consistent with a previous finding that hemangiosarcoma commonly metastasizes to those sites. ${ }^{45}$ Although many older dogs develop hyperplastic liver nodules, our results indicated that in the presence of a splenic mass, a solitary liver nodule is more likely to be metastatic than benign and that if multiple liver nodules are present, the probability that they represent metastases increases slightly.

The present study and the online decision-support calculator derived from it have several potential limitations in addition to those previously mentioned. The model produced by our analysis is just one of many possible models that could aid in distinguishing benign from malignant splenic masses prior to surgery, as demonstrated by the finding that only 1 variable, STP concentration, was common to the previously described model ${ }^{33}$ and the model of the present study. An important difference between the 2 models is that the earlier model estimates the probability of splenic hemangiosarcoma in dogs with nontraumatic hemoabdomen of any cause, whereas our model estimates the probability that a known splenic mass is malignant regardless of whether hemoabdomen is present. Histologic slide reviews and ultrasonographic image interpretations in our study were each performed by 1 investigator, although both are subject to interobserver variability. Assessment of the degree of this variability, as well as reclassification of splenic fibrohistiocytic nodules into other benign or malignant diseases by immunohistochemical methods, ${ }^{46}$ was beyond the scope of the study. Minor inconsistencies in positioning of dogs for ultrasonographic examinations and the use of imaging reports to aid in the assessment of ultrasonographic variables for a small proportion of dogs may have affected our results. Several vari- ables that were significantly associated with malignancy in our univariable analysis were not considered in multivariable analysis because substantial proportions of the study dogs did not have data available for these variables. Inclusion of these variables would have weakened the power of the multivariable analysis, in which only patients with values for every candidate variable can be considered. In addition, we were concerned that some of these variables may not be routinely assessed in general practice. Some of the omitted variables, particularly coagulation profile data, serum lactate concentration, and presence of acanthocytes, plausibly could have strong associations with malignancy. However, abnormalities in coagulation profiles and serum lactate concentration largely reflect the severity of hemoabdomen, ${ }^{20}$ a variable that was included in the model. Because of the limited available information concerning the value of ultrasonographic findings in discriminating benign from malignant splenic masses, we considered ultrasonographic examination of the abdomen to be an inclusion criterion for the study. This increased the likelihood that ultrasonographic variables would be included in the multivariable analysis if they were significant in univariable analysis and may have biased the study toward including them in the final model. Our study population consisted only of dogs whose owners elected surgery, and because there may be hospital-to-hospital variability in the financial and prognostic factors owners consider in deciding whether to elect surgery, our model may be somewhat specific to hospitals with clientele similar to ours. Although hemangiosarcoma is usually the clinical rule out of greatest concern for dogs with splenic masses, 64 of the 229 (27.9\%) malignant masses considered during model development were tumors other than hemangiosarcoma. However, many of the malignant tumors observed, such as histiocytic sarcoma,${ }^{47}$ are associated with high mortality rates and, like hemangiosarcoma, are unlikely to be cured by splenectomy alone.

\section{Acknowledgments}

No external funding was used in this study. The authors declare that there were no conflicts of interest.

Presented in part at the Veterinary Cancer Society Meeting, Minneapolis, October 2013.

The authors thank Cummings School of Veterinary Medicine students Laurel Bifano, Grace Barnett, Jessie Hamilton, and Stephanie Kozol for assistance with data collection.

\section{Footnotes}

\footnotetext{
a. StringSoft, StringSoft Inc, Nashua, NH.

b. Coag Dx, Idexx Laboratories Corp, Westbrook, Me

c. Start 4, Diagnostica Stago Inc, Asnieres-sur-Seine, France.

d. Cell Marque, Rocklin, Calif.

e. SAS, version 9.4, SAS Institute Inc, Cary, NC.

f. Package rms, version 6.0-1, R Foundation for Statistical Computing, Vienna, Austria.

g. Tufts splenic tumor assessment tool. Available at: T-STAT.net and T-STAT.org. Accessed May 10, 2021.
}

\section{References}

1. Johnson KA, Powers BE, Withrow SJ, et al. Splenomegaly in dogs. Predictors of neoplasia and survival after splenectomy. J Vet Intern Med 1989;3:160-166.

2. Day MJ, Lucke VM, Pearson H. A review of pathological diagnoses made from 87 canine splenic biopsies. J Small Anim Pract 1995;36:426-433. 
3. Spangler WL, Culbertson MR. Prevalence, type, and importance of splenic diseases in dogs: 1,480 cases (1985-1989). J Am Vet Med Assoc 1992;200:829-834.

4. MacVean DW, Monlux AW, Anderson PS Jr, et al. Frequency of canine and feline tumors in a defined population. Vet Pathol 1978;15:700-715.

5. Brown NO, Patnaik AK, MacEwen EG. Canine hemangiosarcoma: retrospective analysis of 104 cases. J Am Vet Med Assoc $1985 ; 186: 56-58$.

6. Kim SE, Liptak JM, Gall TT, et al. Epirubicin in the adjuvant treatment of splenic hemangiosarcoma in dogs: 59 cases (1997-2004). J Am Vet Med Assoc 2007;231:1550-1557.

7. Vail DM, MacEwen EG, Kurzman ID, et al. Liposome-encapsulated muramyl tripeptide phosphtidylethanolamine adjuvant immunotherapy for splenic hemangiosarcoma in the dog: a randomized multi-institutional clinical trial. Clin Cancer Res $1995 ; 1: 1165-1170$.

8. Hammer AS, Couto CG, Filppi J, et al. Efficacy and toxicity of VAC chemotherapy (vincristine, doxorubicin and cyclophosphamide) in dogs with hemangiosarcoma. J Vet Intern Med 1991;5:160-166.

9. Sorenmo KU, Baez JL, Clifford CA, et al. Efficacy and toxicity of a dose-intensified doxorubicin protocol in canine hemangiosarcoma. J Vet Intern Med 2004;18:209-213.

10. Ogilvie GK, Powers BE, Mallinckrodt $\mathrm{CH}$, et al. Surgery and doxorubicin in dogs with hemangiosarcoma. $J$ Vet Intern Med 1996;10:379-384.

11. Wood CA, Moore AS, Gliatto JM, et al. Prognosis for dogs with stage I or II splenic hemangiosarcoma treated by splenectomy alone: 32 cases (1991-1993). J Am Anim Hosp Assoc 1998;34:417-421.

12. Payne SE, Rassnick KM, Northrup NC, et al. Treatment of vascular and soft-tissue sarcomas in dogs using an alternating protocol of ifosfamide and doxorubicin. Vet Comp Oncol 2003;1:171-179.

13. Prymak C, McKee LJ, Goldschmidt MH, et al. Epidemiologic, clinical, pathologic, and prognostic characteristics of splenic hemangiosarcoma and splenic hematoma in dogs: 217 cases (1985). J Am Vet Med Assoc 1988;193:706-712.

14. Wendelburg KM, Price LL, Burgess KE, et al. Survival time of dogs with splenic hemangiosarcoma treated by splenectomy with or without adjunctive chemotherapy: 208 cases (2001-2012). J Am Vet Med Assoc 2015;247:393-403.

15. Spangler WL, Kass PH. Pathologic factors affecting postsplenectomy survival in dogs. J Vet Intern Med 1997;11:166-171.

16. Weinstein MJ, Carpenter JL, Schunk CJ. Nonangiogenic and nonlymphomatous sarcomas of the canine spleen: 57 cases (1975-1987). J Am Vet Med Assoc 1989;195:784-788.

17. Wong RW, Gonsalves MN, Huber ML, et al. Erythrocyte and biochemical abnormalities as diagnostic markers in dogs with hemangiosarcoma related hemoabdomen. Vet Surg 2015;44:852-857.

18. Watson AT, Penninck D, Knoll JS, et al. Safety and correlation of test results of combined ultrasound-guided fine-needle aspiration and needle core biopsy of the canine spleen. Vet Radiol Ultrasound 2011;52:317-322.

19. Bertazzolo W, Dell'Orco M, Bonfanti U, et al. Canine angiosarcoma: cytologic, histologic, and immunohistochemical correlations. Vet Clin Pathol 2005;34:28-34

20. Thamm DH. Hemangiosarcoma. In: Withrow S, Vail D, eds. Withrow and MacEwen's small animal clinical oncology. 4th ed. St Louis: WB Saunders Co, 2007;679-688.

21. Thamm DH, Kamstock DA, Sharp CR, et al. Elevated thymidine kinase activity in canine splenic hemangiosarcoma. Vet Comp Oncol 2012;10:292-302.

22. Kirby GM, Mackay A, Grant A, et al. Concentration of lipocalin region of collagen XXVII alpha 1 in the serum of dogs with hemangiosarcoma. J Vet Intern Med 2011;25:497-503.

23. Wrigley RH, Park RD, Kone LJ, et al. Ultrasonographic features of splenic hemangiosarcoma in dogs: 18 cases (19801986). J Am Vet Med Assoc 1988;192:1113-1117.

24. Cuccovillo A, Lamb CR. Cellular features of sonographic target lesions of the liver and spleen in 21 dogs and a cat. Vet Radiol Ultrasound 2002; 43:275-278

25. Ivancić M, Long F, Seiler GS. Contrast harmonic ultrasonog raphy of splenic masses and associated liver nodules in dogs. J Am Vet Med Assoc 2009;234:88-94.

26. Ohlerth S, Dennler M, Rüefli E, et al. Contrast harmonic imaging characterization of canine splenic lesions. J Vet Intern Med 2008;22:1095-1102.

27. Aronsohn MG, Dubiel B, Roberts B, et al. Prognosis for acute nontraumatic hemoperitoneum in the dog: a retrospective analysis of 60 cases (2003-2006). J Am Anim Hosp Assoc 2009; 45:72-77.

28. Pintar J, Breitschwerdt EB, Hardie EM, et al. Acute nontraumatic hemoabdomen in the dog: a retrospective analysis of 39 cases (1987-2001). J Am Anim Hosp Assoc 2003;39:518-522.

29. Hammond TN, Pesillo-Crosby SA. Prevalence of hemangiosarcoma in anemic dogs with a splenic mass and hemoperitoneum requiring a transfusion: 71 cases (2003-2005). J Am Vet Med Assoc 2008;232:553-558.

30. Mallinckrodt MJ, Gottfried SD. Mass-to-splenic volume ratio and splenic weight as a percentage of body weight in dogs with malignant and benign splenic masses: 65 cases. $J$ Am Vet Med Assoc 2011;239:1325-1327.

31. Kshirsagar AV, Chiu Y-L, Bomback AS, et al. A hypertension risk score for middle-aged and older adults. J Clin Hypertens (Greenwich) 2010;12:800-808

32. Losina E, Klara K, Michl GL, et al. Development and feasibility of a personalized, interactive risk calculator for knee osteoarthritis. BMC Musculoskelet Disord 2015;16:312.

33. Schick AR, Hayes GM, Singh A, et al. Development and validation of a hemangiosarcoma likelihood prediction model in dogs presenting with spontaneous hemoabdomen: the HeLP score. J Vet Emerg Crit Care (San Antonio) 2019;29:239-245.

34. Sehested M, Hou-Jensen K. Factor VIII related antigen as an endothelial cell marker in benign and malignant diseases. Virchows Arch A Pathol Anat Histol 1981;391:217-225.

35. Spangler WL, Kass PH. Pathologic and prognostic characteristics of splenomegaly in dogs due to fibrohistiocytic nodules: 98 cases. Vet Pathol 1998;35:488-498.

36. Hosmer DW, Lemeshow S. Assessing the fit of the model. In: Applied logistic regression. 2nd ed. New York: John Wiley and Sons, 2000;143-202.

37. Ceron JJ, Eckersell PD, Martýnez-Subiela S. Acute phase proteins in dogs and cats: current knowledge and future perspectives. Vet Clin Pathol 2005;34:85-99.

38. Tecles F, Caldín M, Zanella, et al. Serum acute-phase protein concentrations in female dogs with mammary tumors. $J$ Vet Diagn Invest 2009;21:214-219.

39. Tecles F, Spiranelli E, Bonfanti U, et al. Preliminary studies of serum acute-phase protein concentrations in hematologic and neoplastic diseases of the dog. J Vet Intern Med 2005; 19:865-870

40. Warry E, Bohn A, Emanuelli M, et al. Disease distribution in canine patients with acanthocytosis: 123 cases. Vet Clin Pathol 2013;42:465-470.

41. Hirsch VM, Jacobsen J. Mills JH. A retrospective study of canine hemangiosarcoma and its association with acanthocytosis. Can VetJ 1981;22:152-155.

42. Tant MS, Lumsden JH, Jacobs RM, et al. Evaluation of acanthocyte count as a diagnostic test for canine hemangiosarcoma. Comp Clin Patbol 2004;12:174-178.

43. Hammer AS, Couto CG, Swardson C, et al. Hemostatic abnormalities in dogs with hemangiosarcoma. $J$ Vet Intern Med 1991;5:11-14.

44. Levinson JG, Bouma JL, Althouse GC, et al. Prevalence of malignancy when solitary versus multiple lesions are detected during abdominal ultrasonographic examination of dogs with spontaneous hemoperitoneum: 31 cases (2003-2008). $J$ Vet Emerg Crit Care (San Antonio) 2009;19:496-500.

45. Waters DJ, Caywood DD, Hayden DW, et al. Metastatic patterns in dogs with splenic hemangiosarcoma: clinical implications. J Small Anim Pract 1988;29:805-814.

46. Moore AS, Frimberger AE, Sullivan N, et al. Histologic and immunohistochemical review of splenic fibrohistiocytic nodules in dogs. J Vet Intern Med 2012;26:1164-1168.

47. Skorupski KA, Clifford CA, Paolini MC, et al. CCNU for the treatment of dogs with histiocytic sarcoma.J Vet Intern Med 2007;21:121-126. 